\title{
Approximation of Convex Figures by Pairs of Rectangles*
}

\author{
Otfried Schwarzkopf ${ }^{\dagger} \quad$ Ulrich Fuchs ${ }^{\ddagger} \quad$ Günter Rote R $^{\S}$ \\ Emo Welzl ${ }^{\top}$
}

\begin{abstract}
We consider the problem of approximating a convex figure in the plane by a pair $(r, R)$ of homothetic (that is, similar and parallel) rectangles with $r \subseteq C \subseteq R$. We show the existence of such a pair where the sides of the outer rectangle are at most twice as long as the sides of the inner rectangle, thereby solving a problem posed by Pólya and Szegö.

If the $n$ vertices of a convex polygon $C$ are given as a sorted array, such an approximating pair of rectangles can be computed in time $O\left(\log ^{2} n\right)$.
\end{abstract}

\section{Introduction}

Let $C$ be a convex figure in the plane. A pair of rectangles $(r, R)$ is called an approximating pair for $C$, if $r \subseteq C \subseteq R$ and if $r$ and $R$ are homothetic, that is, they are parallel and have the same aspect ratio. Note that this is equivalent to the existence of an expansion $x \mapsto \lambda\left(x-x_{0}\right)+x_{0}$ (with center $x_{0}$ and expansion factor $\lambda$ ) which maps $r$ into $R$.

We measure the quality $\lambda(r, R)$ of our approximating pair $(r, R)$ as the quotient of the length of a side of $R$ divided by the length of the corresponding side of $r$. This is just the expansion factor $\lambda$ used in the above expansion mapping.

The motivation for our investigation is the use of $r$ and $R$ as simple certificates for the impossibility or possibility of obstacle-avoiding motions of $C$. If $R$ can be moved along a path without hitting a given set of obstacles, then this is also possible for $C$. Let's say that a motion planning problem for $C$ is simple if a motion is still possible for $C$ expanded by a factor of 2 . Now, if $(r, R)$ has quality 2 , then every simple motion planning problem for $C$ has also a solution for $R$. More details can be found in $\left[\mathrm{FMR}^{+} 90\right]$.

Pólya and Szegő [PS51] showed that for every convex figure $C$ there is an approximating pair $(r, R)$ with $\lambda(r, R) \leq 3$, and raised the question whether this upper bound could be improved. In fact, an improvement to $2 \sqrt{2}$ follows from work of John [Joh48] and Leichtweiß [Lei59]. They proved that for every convex figure $C$ in the plane there is an approximating pair of homothetic ellipses with quality 2 . Since any ellipse has an approximating pair of rectangles with quality $\sqrt{2}$, the claimed bound of $2 \sqrt{2}$ follows. A related problem has been considered by Lassak [Las89],

\footnotetext{
*This research was supported by the Deutsche Forschungsgemeinschaft under Grant Al 253/11, Schwerpunktprogramm "Datenstrukturen und effiziente Algorithmen", and by the ESPRIT II Basic Research Action of the European Community under contract No. 3075 (project ALCOM). It was done while the authors were at the Freie Universität Berlin.

${ }^{\dagger}$ Department of Computer Science, Postech, Hyoja-dong, Pohang 790-784, South Korea

$\ddagger$ Fachbereich Mathematik, Freie Universität Berlin, Arnimallee 2-6, D-15195 Berlin, Germany

$\S$ Institut für Mathematik, Technische Universität Graz, Steyrergasse 20, A-8010 Graz, Austria

IDepartement Informatik, ETH Zürich, IFW, ETH Zentrum, CH-8092 Zürich, Switzerland
} 
who showed that for every centrally symmetric convex body $M$ and every (not necessarily centrally symmetric) convex figure $C$, there are two concentric affine images $a$ and $A$ of $M$ with $a \subseteq C \subseteq A$. He proved that the expansion factor between $a$ and $A$ can always be chosen to be $\sqrt{2}+1$, which is optimal.

The question of further improvement in our problem has remained open. In the present paper we settle the problem by demonstrating that for every convex figure there exists an approximating pair $(r, R)$ with a factor $\lambda(r, R) \leq 2$. This bound is optimal, since for a triangle there is no approximating pair with a factor less than 2. (This can be seen by comparing the areas of a minimum circumscribed and a maximum inscribed rectangle for a triangle.) After the proceedings version of this paper [SFR ${ }^{+90]}$ appeared, this result has been independently obtained by Lassak [Las93], using the same basic idea. In contrast, our proof of Lemma 7 is more geometric in nature than the corresponding proof in [Las93], and in addition, we show how to find the approximating pair efficiently.

Many problems about inner and outer approximation by homothetic figures remain open. For example, the optimal quality bound for an approximating pair of homothetic triangles is not known exactly. Fleischer, Mehlhorn, Rote, Welzl, and Yap $\left[\mathrm{FMR}^{+} 90\right]$ showed that it lies between $1+\sqrt{5} / 2 \approx 2.118$ and 2.25 ; see also Lassak [Las92] for a related result.

In Section 2 we consider approximations by rectangles with a fixed orientation. On the one hand, this prepares the basics for the upper bound, and on the other hand we show that an optimal approximating pair with a fixed orientation can be computed in time $O(\log n)$ if $C$ is a convex $n$-gon whose vertices are stored in a sorted array. The algorithm is an application of the tentative-prune-and-search technique of Kirkpatrick and Snoeyink [KS95]. In Section 3 we show the existence of approximating pairs of quality 2 and in Section 4 we present an algorithm which computes such a pair in time $O\left(\log ^{2} n\right)$.

This paper is an improved version of our conference paper [SFR ${ }^{+90]}$. The algorithms there were slower by a factor of $O(\log n)$ because the tentative-prune-andsearch technique was not available and we had to use nested binary search instead.

\section{Approximation with a Fixed Orientation}

Let $r$ be a rectangle with a counterclockwise numbering $v_{1}(r), v_{2}(r), v_{3}(r), v_{4}(r)$ of its vertices. The orientation $\alpha(r)$ is the directed angle between the positive $x$-axis and the vector from $v_{1}(r)$ to $v_{2}(r)$, and the aspect ratio is

$$
\sigma(r)=\frac{\left|v_{2}(r) v_{3}(r)\right|}{\left|v_{1}(r) v_{2}(r)\right|}
$$

$(|p q|$ denotes the distance between $p$ and $q)$. By $\mathcal{R}(\alpha, \sigma)$ we denote the set of all rectangles with orientation $\alpha$ and aspect ratio $\sigma$, see Figure 1 .

Note that, depending on the choice of the vertex $v_{1}$, a rectangle belongs to the classes $\mathcal{R}(\alpha, \sigma), \mathcal{R}\left(\alpha+\frac{\pi}{2}, \frac{1}{\sigma}\right), \mathcal{R}(\alpha+\pi, \sigma)$, or $\mathcal{R}\left(\alpha+\frac{3 \pi}{2}, \frac{1}{\sigma}\right)$. For the time being, whenever we talk about a rectangle, we assume that we have a fixed counterclockwise numbering of the vertices. Let $C$ denote a bounded convex figure in the plane. For every $\alpha$ there is a unique minimum area rectangle $R(\alpha)$ with orientation $\alpha$ enclosing $C$. Let $\sigma(\alpha)$ denote the aspect ratio of $R(\alpha)$; so $R(\alpha) \in \mathcal{R}(\alpha, \sigma(\alpha))$. $\sigma(\alpha)$ is the quotient of the widths of $C$ when seen from directions $\alpha+\pi / 2$ and $\alpha$. Since the width is a continuous function of $\alpha$ and it is bounded away from $0, \sigma(\alpha)$ is continuous, bounded from above, and bounded away from 0 .

Now consider an approximating pair $(r, R)$ for $C$ with orientation $\alpha . R$ contains the minimum area enclosing rectangle $R(\alpha)$. Since we can shrink $r$ and $R$ appropriately, we may as well assume that $R=R(\alpha)$, so $r \in \mathcal{R}(\alpha, \sigma(\alpha))$. The problem of 


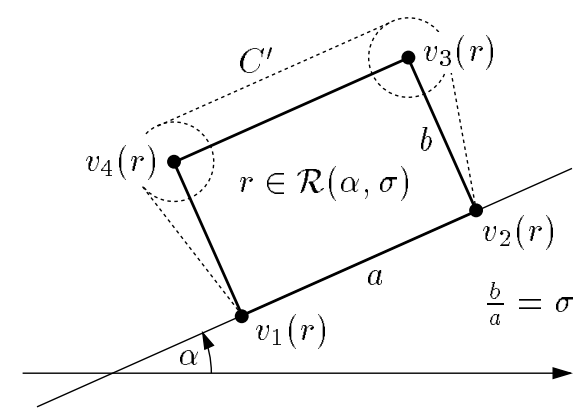

Figure 1: A rectangle with orientation $\alpha$ and aspect ratio $\sigma$

finding the best approximating pair with orientation $\alpha$ thus reduces to the problem of finding the largest rectangle with orientation $\alpha$ and aspect ratio $\sigma(\alpha)$ contained in $C$. If we define

$$
\mathcal{F}_{0}(\alpha):=\{r \in \mathcal{R}(\alpha, \sigma(\alpha)) \mid r \subseteq C\},
$$

the problem becomes: Find the largest rectangle in $\mathcal{F}_{0}(\alpha)$. We define $\mathcal{F}(\alpha)$ as the set of largest rectangles in $\mathcal{F}_{0}(\alpha)$. We have

Lemma 1 Let $r$ be a rectangle in $\mathcal{F}(\alpha)$. Then two diagonal vertices of $r$ lie on the boundary $\partial C$ of $C$.

Proof: If at most one vertex of $r$ lies on $\partial C, r$ is clearly not maximal. So assume vertices $v_{1}(r)$ and $v_{2}(r)$ lie on $\partial C$, while $v_{3}(r)$ and $v_{4}(r)$ don't, see Figure 1 . Then there are two disks $U_{3}, U_{4}$ around $v_{3}(r)$ and $v_{4}(r)$ which are contained in $C$. By convexity of $C$, the convex hull $C^{\prime}$ of $v_{1}(r), v_{2}(r), U_{3}$, and $U_{4}$ is contained in $C$. But there is a larger copy of $r$ in $C^{\prime}$, contradicting the maximality of $r$.

Lemma 2 The side lengths of all rectangles $r \in \mathcal{F}(\alpha)$, for all $\alpha$, are uniformly bounded from above and bounded away from zero.

Proof: Since every rectangle $r$ is contained in $C$, an upper bound is trivial. The function $\sigma(\alpha)$ is bounded from above and bounded away from zero. Therefore, the shortest side of the rectangle with aspect ratio $\sigma(\alpha)$ which is contained in the incircle of $C$ is bounded away from zero. This is a lower bound for the shortest side of any rectangle $r \in \mathcal{F}(\alpha)$.

We give an algorithm that computes the largest rectangle with fixed orientation and shape contained in a polygon with $n$ vertices in time $O(\log n)$. Since $\sigma(\alpha)$ can be computed in time $O(\log n)$ using standard search techniques, the best approximating pair with fixed orientation $\alpha$ can be computed in the same time.

We remark that a similar problem has be recently treated by Alt, Hsu, and Snoeyink [AHS95]. They compute the largest-area or the largest-perimeter rectangle with a given orientation contained in a polygon with $n$ vertices in time $O(\log n)$. Since there is no restriction on the shape, this problem is more difficult than ours.

Since the following treatment does not use the fact that $R=R(\alpha)$ is a rectangle, we formulate it for an arbitrary convex polygon $A$ instead of $R$.

Lemma 3 Consider a largest positively homothetic copy $A^{\prime}=f(A)$ of a given convex $m$-gon $A=v_{1} v_{2} \ldots v_{m}$ which is contained in a convex figure $C$. Let $f(x)=$ $\lambda\left(x-x_{0}\right)+x_{0}$ denote the homothety with expansion factor $\lambda>0$ mapping $A$ to $A^{\prime}$. Then there are three vertices $v_{i}, v_{j}$, and $v_{k}$ of $A$ such that the triangle $f\left(v_{i} v_{j} v_{k}\right)$ is a largest positively homothetic copy of the triangle $v_{i} v_{j} v_{k}$ contained in $C$. 
Proof: The problem at hand can be formulated as an optimization problem as follows.

$$
\begin{array}{ll}
\text { maximize } & \lambda \\
\text { subject to } & f\left(v_{i}\right)=\lambda\left(v_{i}-x_{0}\right)+x_{0} \in C, \quad \text { for } i=1, \ldots, m \\
& \lambda \in \mathbf{R}, x_{0} \in \mathbf{R}^{2}
\end{array}
$$

This is a convex programming problem in three variables with a linear objective function and $m$ convex constraints. It follows from the theory of convex programming, or, if you will, from Helly's theorem, that there is a subset of at most three constraints for which the corresponding problem has the same optimal value of $\lambda$ [A94]. In geometrical terms, the problem with the reduced subset of three constraints describes the largest homothetic triangle.

This lemma suggests a way to reduce the problem of finding the largest homothetic copy of a convex $m$-gon $A$ in $C$ to $\left(\begin{array}{c}m \\ 3\end{array}\right)$ problems of finding the largest homothetic copy of a triangle contained in $C$ : For each triple of vertices $v_{i} v_{j} v_{k}$, find the largest homothetic triangle contained in $C$. The smallest of the $\left(\begin{array}{c}m \\ 3\end{array}\right)$ expansion factors is the expansion factor for $A$.

In the end, if we want to actually find the optimal homothety $f$ which has $f(A) \subseteq C$, we encounter a slight technical problem because the largest homothetic triangle $t \subseteq C$ need not be unique. However, in this case there must be two parallel sides of $C$ along which $t$ can slide. So we may have to slide the corresponding placements $f(A)$ along a given direction in order to find the correct position inside $C$. This amounts to the following problem.

Given an $m$-gon $A_{0}$, an $n$-gon $C$, and a direction $x$, find a value $\beta \in \mathbf{R}$ such that the copy of $A_{0}$ translated by $\beta x$ is contained in $C$.

The polygon $A_{0}$ is $f(A)$ for some homothety $f$ that is optimal for three vertices $v_{i} v_{j} v_{k}$. The problem can be solved in $O(m \log n)$ time as follows:

1. For each vertex $v$ of $A_{0}$, intersect the line parallel to $x$ through $v$ with $C$, in $O(\log n)$ time. These intersection points determine an interval of values $\beta$ for which $v+\beta x \in C$.

2. Intersect the $m$ intervals. Any value $\beta$ in the intersection corresponds to a translation of $A_{0}$ for which all vertices are contained in $C$, and therefore, the polygon itself is contained in $C$.

Each of the largest homothetic triangle problems can be solved in $O(\log n)$ time by the tentative-prune-and-search technique of Kirkpatrick and Snoeyink [KS95]. Summarizing, we have the following lemma.

Lemma 4 Given two polygons $A$ and $C$ with $m$ and $n$ vertices, the largest homothetic copy of $A$ which can be placed in $C$ can be computed in time $O\left(m^{3} \log n\right)$.

Of course, this is only a good approach when $m$ is small. When $m$ is larger, the problem can be solved in $O(m+n)$ time as a linear programming problem in three variables. In our case of rectangles, we have $m=4$ and thus we get the following theorem.

Theorem 5 Given a convex polygon with $n$ vertices in the plane, the optimal approximating pair of rectangles with a fixed orientation $\alpha$ can be computed in time $O(\log n)$. 


\section{The Upper Bound on the Approximation Ratio}

We now solve the problem posed by Pólya and Szegö.

Theorem 6 For every convex figure there exists an approximating pair $(r, R)$ of rectangles with $\lambda(r, R) \leq 2$.

In a first step we show that if we consider an approximating pair $(r, R(\alpha))$ where all four vertices of $r$ touch the boundary of $C$, we have $\lambda(r, R(\alpha)) \leq 2$. Using a continuity argument we then show that such a pair always exists.

Lemma 7 Let $q=P Q R S$ be a quadrilateral with inscribed rectangle $A B C D$. If every vertex of $A B C D$ touches $q$ as in Figure 2 then one of the following holds:

$$
a^{\prime} \leq 2 a \quad \text { or } \quad b^{\prime} \leq 2 b .
$$

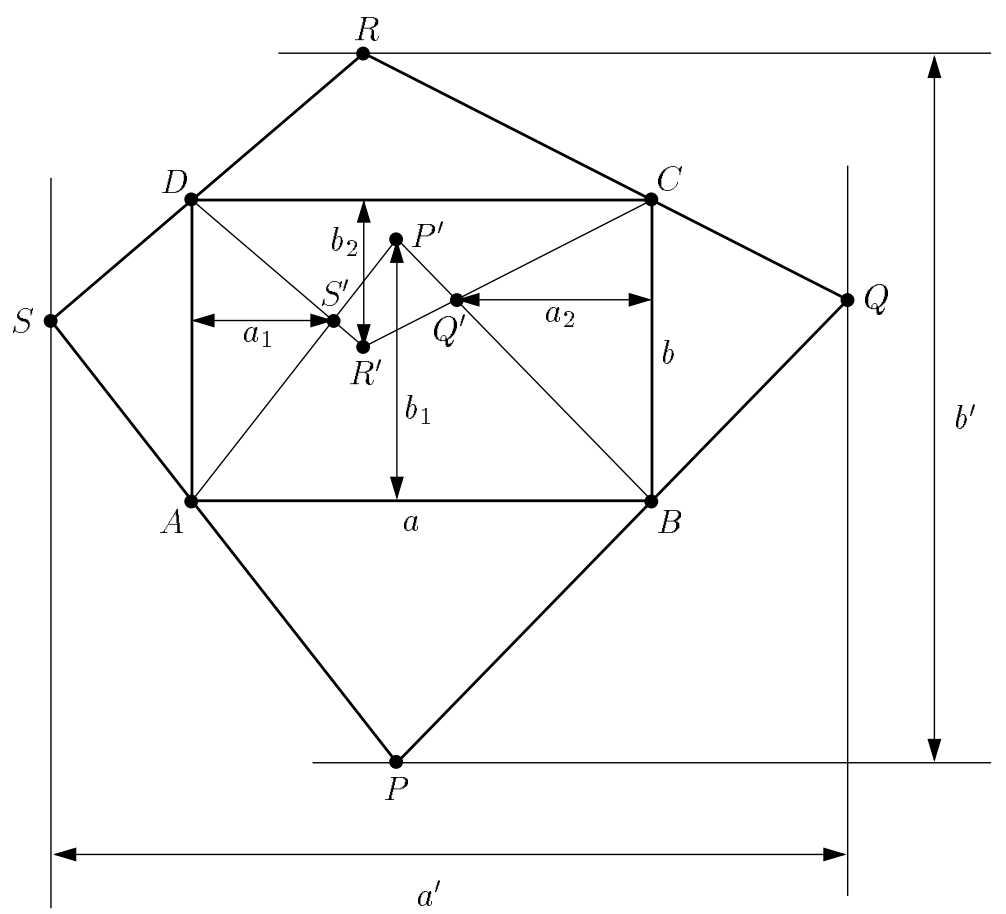

Figure 2: A rectangle inscribed in a quadrilateral

Proof: We reflect $P$ at $A B$ to obtain $P^{\prime}, Q$ at $B C$ etc. In other words, we "wrap" a sheet with the shape of $q$ around the rectangle $A B C D$. (We may think of the rectangle as a piece of chocolate.) We have to show that either $a_{1}+a_{2} \leq a$ or $b_{1}+b_{2} \leq b$.

There are two cases: If the triangles $A B P^{\prime}, B C Q^{\prime}, C D R^{\prime}, D A S^{\prime}$ do not overlap, their area is less than the area of $A B C D$, so we have $\frac{1}{2}\left(a_{2} b+b_{2} a+a_{1} b+b_{1} a\right)=$ $\frac{1}{2}\left(\left(a_{1}+a_{2}\right) b+a\left(b_{1}+b_{2}\right)\right) \leq a b$. This is impossible for $a_{1}+a_{2}>a$ and $b_{1}+b_{2}>b$.

So assume without loss of generality that $A B P^{\prime}$ and $C D R^{\prime}$ overlap, as depicted in Figure 2. Then we can cut $A B C D$ vertically through any point in the intersection of $A B P^{\prime}$ and $C D R^{\prime}$. $S^{\prime}$ lies to the left, $Q^{\prime}$ to the right of this cut. This implies $a_{1}+a_{2} \leq a$. 
Lassak [Las93] has given a more general statement: Under the conditions and with the notations of the previous lemma, we have

$$
\frac{a}{a^{\prime}}+\frac{b}{b^{\prime}} \geq 1 \text {. }
$$

Lassak proved this by a tedious calculation. He posed as an open problem the generalization to $d>2$ dimensions, where the rectangle is replaced by a rectangular box, $q$ is replaced by a polytope with $2^{d}$ facets, and the left side of the above inequality is a sum of $d$ fractions.

Lemma 8 Let $C$ be a convex figure with an approximating pair $(r, R)$ such that

(i) every vertex of $r$ touches the boundary of $C$, and

(ii) every edge of $R$ touches $C$.

Then $\lambda(r, R) \leq 2$.

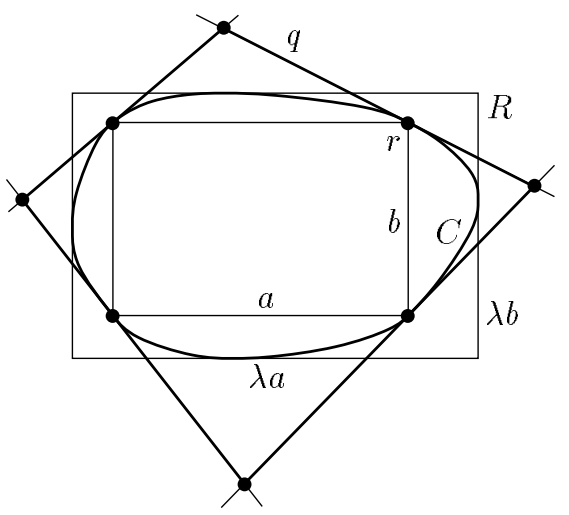

Figure 3: An approximating pair $(r, R)$ for a curve $C$

Proof: Introduce supporting lines for $C$ in every vertex of $r$. They form a quadrilateral $q$ which contains $C$ as in Figure 3. Denote the side lengths of $r$ by $a$ and $b$, and the corresponding side lengths of $R$ by $\lambda a$ and $\lambda b$. Since $C \subseteq q$ and because of (i) and (ii) $\lambda a \leq 2 a$ or $\lambda b \leq 2 b$ holds due to Lemma 7. But in any case this implies $\lambda(r, R)=\lambda \leq 2$.

Thus, in order to prove Theorem 6 we have to find a direction $\alpha$ and an approximating pair of rectangles fulfilling the conditions of Lemma 8 . The idea of the proof is to consider, for each direction $\alpha$, the unique smallest rectangle $R(\alpha)$ enclosing $C$, and the largest inscribed homothetic copy $r(\alpha)$ of $R(\alpha)$, as in Section 2. By Lemma 1, $r(\alpha)$ must touch $\partial C$ at two diagonal vertices, either $v_{1}$ and $v_{3}$ (case 1 ) or at $v_{2}$ and $v_{4}$ (case 2). If we rotate $\alpha$ from 0 to $\pi / 2$, cases 1 and 2 exchange roles. Intuitively, there must be an intermediate direction where the situation changes and all four vertices touch $\partial C$.

To make these ideas and the underlying continuity arguments precise requires a little more formal work, mainly to cope with degeneracies, since the placement of $r(\alpha)$ need not be unique.

Lemma 9 Let $r^{\prime}, r^{\prime \prime} \in \mathcal{F}(\alpha)$ with $\left\{v_{1}\left(r^{\prime}\right), v_{3}\left(r^{\prime}\right)\right\} \subset \partial C$ and $\left\{v_{2}\left(r^{\prime \prime}\right), v_{4}\left(r^{\prime \prime}\right)\right\} \subset \partial C$. Then

$$
\left\{v_{1}\left(r^{\prime}\right), v_{2}\left(r^{\prime}\right), v_{3}\left(r^{\prime}\right), v_{4}\left(r^{\prime}\right)\right\} \subset \partial C \text { and }\left\{v_{1}\left(r^{\prime \prime}\right), v_{2}\left(r^{\prime \prime}\right), v_{3}\left(r^{\prime \prime}\right), v_{4}\left(r^{\prime \prime}\right)\right\} \subset \partial C .
$$


Proof: If $r^{\prime}=r^{\prime \prime}$ there is nothing to prove. So we assume that there is a translation transforming $r^{\prime}$ into $r^{\prime \prime}$.

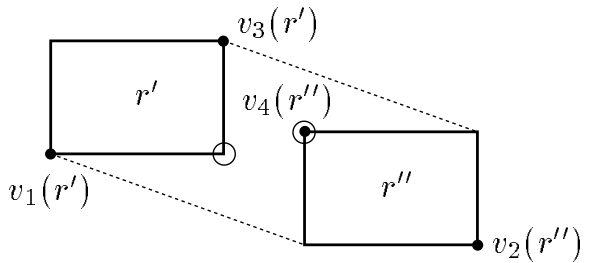

(a)

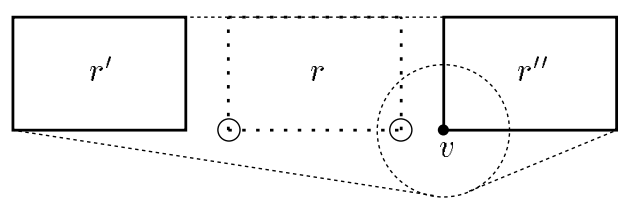

(b)

Figure 4: The proof of Lemma 9

The case that the translation direction is not parallel to any edge of $r^{\prime}, r^{\prime \prime}$ (Figure 4a) is impossible, because the two encircled vertices in Figure 4a would lie in the interior of the convex hull of $r^{\prime}$ and $r^{\prime \prime}$, hence in the interior of $C$. This contradicts the assumption of the lemma.

Therefore, the translation direction must be parallel to an edge of $r^{\prime}$ and $r^{\prime \prime}$, as shown in Figure 4b. Let $r \in \mathcal{F}(\alpha)$ be the rectangle half-way between $r^{\prime}$ and $r^{\prime \prime}$ and of the same size. Suppose that some vertex $v$ of $r^{\prime}$ or $r^{\prime \prime}$ does not lie on $\partial C$. Consider a disk around the vertex $v$ contained in $C$, and take the convex hull of this disk, $r^{\prime}$, and $r^{\prime \prime}$, see Figure $4 \mathrm{~b}$. There is an edge of $r$ that lies in the interior of this convex hull and hence does not touch $\partial C$. This contradicts Lemma 1 . Therefore, all vertices of $r^{\prime}$ and $r^{\prime \prime}$ (and $r$ ) lie on $\partial C$.

Proof: (of Theorem 6) We define

$$
\begin{aligned}
& A_{1}:=\left\{\alpha \in\left[0 \ldots \frac{\pi}{2}\right] \mid \exists r \in \mathcal{F}(\alpha) \text { with }\left\{v_{1}(r), v_{3}(r)\right\} \subset \partial C\right\} \text { and } \\
& A_{2}:=\left\{\alpha \in\left[0 \ldots \frac{\pi}{2}\right] \mid \exists r \in \mathcal{F}(\alpha) \text { with }\left\{v_{2}(r), v_{4}(r)\right\} \subset \partial C\right\} .
\end{aligned}
$$

From Lemma 1 it follows that $A_{1} \cup A_{2}=\left[0 \ldots \frac{\pi}{2}\right]$. Since $\sigma(\pi / 2)=1 / \sigma(0), \mathcal{F}(0)$ and $\mathcal{F}\left(\frac{\pi}{2}\right)$ contain the same rectangles. By definition we have

$$
\begin{aligned}
& 0 \in A_{1} \quad \Longrightarrow \quad \frac{\pi}{2} \in A_{2} \\
& 0 \in A_{2} \quad \Longrightarrow \quad \frac{\pi}{2} \in A_{1}
\end{aligned}
$$

and so $A_{1} \neq \emptyset, A_{2} \neq \emptyset$. We will soon show that $A_{1}$ and $A_{2}$ are closed sets. Since $\left[0 \ldots \frac{\pi}{2}\right]$ is connected and $A_{1} \cup A_{2}=\left[0 \ldots \frac{\pi}{2}\right]$, the two sets must intersect. Let $\alpha \in A_{1} \cap A_{2}$. This means that there are two rectangles $r^{\prime}, r^{\prime \prime} \in \mathcal{F}(\alpha)$ with $\left\{v_{1}\left(r^{\prime}\right), v_{3}\left(r^{\prime}\right), v_{2}\left(r^{\prime \prime}\right), v_{4}\left(r^{\prime \prime}\right)\right\} \subset \partial C$.

By Lemma 9, the rectangle $r^{\prime}$ has all four vertices on the boundary of $C$. Together with the smallest circumscribed rectangle $R$ with orientation $\alpha$, it forms an approximating pair $\left(r^{\prime}, R\right)$. By Lemma 8 , we have $\lambda\left(r^{\prime}, R\right) \leq 2$, and we are done.

It remains to show that the sets $A_{1}$ and $A_{2}$ are closed. Because of symmetry we consider $A_{1}$ only. Let $\left(\alpha_{1}, \alpha_{2}, \ldots\right)$ be a sequence with $\alpha_{i} \in A_{1}$ and $\lim _{i \rightarrow \infty} \alpha_{i}=\alpha$. We want to show that $\alpha \in A_{1}$. For every $i$, choose a rectangle $r_{i} \in \mathcal{F}\left(\alpha_{i}\right)$ with $\left\{v_{1}\left(r_{i}\right), v_{3}\left(r_{i}\right)\right\} \subset \partial C$. By the theorem of Bolzano and Weierstraß we can select a subsequence of rectangles such that their lower left vertices $v_{1}$ converge. Repeating this, we can select a subsequence in which the vertices $v_{2}$ converge, too. By change of notation we denote this subsequence again by $\left(\alpha_{i}\right)$. Since $v_{3}$ and $v_{4}$ depend continuously on $\alpha, v_{1}$, and $v_{2}$, the sequences $v_{3}\left(r_{i}\right)$ and $v_{4}\left(r_{i}\right)$ also converge. Let's denote

$$
v_{j}:=\lim _{i \rightarrow \infty} v_{j}\left(r_{i}\right) \text {, for } j=1,2,3,4 \text {. }
$$


We claim that the quadrilateral with vertices $v_{1} v_{2} v_{3} v_{4}$ is in $\mathcal{F}(\alpha)$.

By Lemma 2, the distances between any two vertices of a rectangle $r_{i}$ are bounded away from zero. Therefore the four limit vertices are distinct, and the orientations and lengths of the sides of $r_{i}$, as well as the aspect ratios, converge. Since the orientations of the sides can only converge to $\alpha$ and $\alpha+\frac{\pi}{2}, r$ is indeed a rectangle. The limit of the aspect ratios is

$$
\lim _{i \rightarrow \infty} \sigma\left(r_{i}\right)=\lim _{i \rightarrow \infty} \sigma\left(\alpha_{i}\right)=\sigma(\alpha),
$$

since $\sigma$ is continuous. Since $\partial C$ is closed, $\left\{v_{1}, v_{3}\right\} \subset \partial C$, and hence $r \in \mathcal{F}_{0}(\alpha)$.

We still have to show that $r \in \mathcal{F}(\alpha)$, or in other words, that $r$ is maximal. Assume the contrary, and let $\tilde{r} \in \mathcal{F}(\alpha)$ be a rectangle which is larger than $r$ by a factor $1+\varepsilon$, for some $\varepsilon>0$. Now, consider a translated copy $r^{\prime}$ of the rectangle $(1+\varepsilon / 2) r$ which is placed inside $\tilde{r}$ and concentric with $\tilde{r}$. There is some angle $\beta_{0}>0$ such that $r^{\prime}$ can be rotated around its center by any angle $\beta$ with $|\beta| \leq \beta_{0}$ and still be contained in $\tilde{r}$.

In the sequence of rectangles which converge to $r$, there must be a rectangle $r_{i} \in \mathcal{F}\left(\alpha_{i}\right)$ with $\left|\alpha_{i}-\alpha\right| \leq \beta_{0}$ and $\left|v_{1}\left(r_{i}\right) v_{2}\left(r_{i}\right)\right| \leq(1+\varepsilon / 3)\left|v_{1} v_{2}\right|,\left|v_{2}\left(r_{i}\right) v_{3}\left(r_{i}\right)\right| \leq$ $(1+\varepsilon / 3)\left|v_{2} v_{3}\right|$. This means that $r_{i}$ can be enlarged by a factor $\frac{1+\varepsilon / 2}{1+\varepsilon / 3}$ and still fit inside $\tilde{r} \subseteq C$, contradicting its maximality.

\section{Finding an Approximating Pair}

Theorem 10 An approximating pair $(r, R)$ of rectangles with $\lambda(r, R) \leq 2$ for a convex polygon $C$ with $n$ vertices given in sorted order can be computed in time $O\left(\log ^{2} n\right)$.

Proof: From the last section we know that for every convex polygon $C$ there is at least one orientation $\alpha_{0}$ such that the largest rectangle in $\mathcal{F}\left(\alpha_{0}\right)$ touches $\partial C$ with all four vertices. We search for $\alpha_{0}$ by binary search on the half-open interval $\left[0 \ldots \frac{\pi}{2}\right)$. First we find the best approximation for orientation 0 using the algorithm of Section 2. We check whether $0 \in A_{1}$ and whether $0 \in A_{2}$. If $0 \in A_{1} \cap A_{2}$ we are done as in Theorem 6 . So we assume that $0 \in A_{1} \backslash A_{2}$. Now we test another orientation $\alpha \in\left[0 \ldots \frac{\pi}{2}\right)$. If $\alpha \in A_{1}$, we continue the search in the interval $\left[\alpha \ldots \frac{\pi}{2}\right)$, otherwise in $[0 \ldots \alpha)$.

We use a discrete set of search orientations: Let $P, Q, R, S$ be the bottommost, rightmost, topmost, and leftmost point of $C$. Consider the orientations of the edges between $P$ and $Q$. Since $C$ is convex, the sequence of orientations of these edges is strictly increasing, so we can perform binary search on them. After $O(\log n)$ tests we have found two adjacent edges with orientations $\alpha^{\prime}, \alpha^{\prime \prime}$ and we know that $\alpha_{0} \in\left[\alpha^{\prime} \ldots \alpha^{\prime \prime}\right)$. Now consider the edges between $Q$ and $R$. We continue the binary search on the set

$$
\left\{\alpha-\frac{\pi}{2} \mid \alpha \text { is the orientation of an edge between } Q \text { and } R\right\} \cap\left[\alpha^{\prime} \ldots \alpha^{\prime \prime}\right) .
$$

In the same manner we proceed with the orientations of the edges between $R$ and $S$ and between $S$ and $P$.

Every test takes time $O(\log n)$ by Theorem 5. Since there are $O(\log n)$ tests, after time $O\left(\log ^{2} n\right)$ we have found orientations $\alpha_{1}, \alpha_{2}$. There are no edges of the polygon with orientation between $\alpha_{1}$ and $\alpha_{2}$, so the enclosing rectangles $R\left(\alpha_{1}\right)$ and $R\left(\alpha_{2}\right)$ touch $C$ in the same four vertices $V_{1}, V_{2}, V_{3}, V_{4}$. Let $r_{1}, r_{2}$ be the largest rectangle in $\mathcal{F}\left(\alpha_{1}\right), \mathcal{F}\left(\alpha_{2}\right)$ resp. By assumption, $\alpha_{1} \in A_{1}, \alpha_{2} \in A_{2}$, so $\left\{v_{1}\left(r_{1}\right), v_{3}\left(r_{1}\right), v_{2}\left(r_{2}\right), v_{4}\left(r_{2}\right)\right\} \subset \partial C$. Let $C^{\prime}$ be the convex hull of $r_{1}, r_{2}$, and $V_{1}$, 
$V_{2}, V_{3}, V_{4}$. Clearly we have $C^{\prime} \subseteq C$ and $\left\{v_{1}\left(r_{1}\right), v_{3}\left(r_{1}\right), v_{2}\left(r_{2}\right), v_{4}\left(r_{2}\right)\right\} \subset \partial C^{\prime}$. But now we have reduced the problem to a problem of constant size. $C^{\prime}$ is a polygon with at most 12 vertices, $R\left(\alpha_{1}\right)$ and $R\left(\alpha_{2}\right)$ are the minimum circumscribed rectangles with orientations $\alpha_{1}, \alpha_{2}$, resp., and we still have $\alpha_{1} \in A_{1}, \alpha_{2} \in A_{2}$, where $A_{1}, A_{2}$ are defined with respect to $C^{\prime}$. As before there is some $\alpha_{0} \in\left[\alpha_{1} \ldots \alpha_{2}\right)$ such that there exists an approximating pair $(r, R)$ for $C^{\prime}$ with $\lambda(r, R) \leq 2$.

Since $C^{\prime} \subseteq C$, we have $r \subseteq C^{\prime} \subseteq C$. Since $\alpha_{0} \in\left[\alpha_{1} \ldots \alpha_{2}\right), R$ is determined by the four vertices $V_{1}, V_{2}, V_{3}, V_{4}$, so we also have $R \supseteq C$. This implies that $(r, R)$ is also an approximating pair for $C$.

It remains to solve the problem for the constant size case. It can be reduced to a constant number of problems of the following type: Let $V_{i}, i \leq 4$, be four points and $\ell_{i}, i \leq 4$, be four lines in the plane. Find a rectangle with vertices $v_{i} \in \ell_{i}$ such that there is a homothetic rectangle with edges $e_{i} \ni V_{i}$. If we select two points $v_{1} \in \ell_{1}$ and $v_{2} \in \ell_{2}$, they must satisfy two conditions: (i) They can be completed to a rectangle with vertices on the other two lines; and (ii) the aspect ratio is equal to the aspect ratio of the parallel rectangle through the four outer points $V_{i}$. Parameterizing $v_{1} \in \ell_{1}$ and $v_{2} \in \ell_{2}$ in the natural way, it is straightforward to write these two conditions as two rational equations in two variables. This leads to a polynomial equation of bounded degree. We leave the details to the reader.

\section{Conclusion}

We have shown that for any convex figure $C$ in the plane there exists an approximating pair of homothetic inscribed and circumscribed rectangles with quality (ratio) at most 2. Moreover, we have given an $O\left(\log ^{2} n\right)$ algorithm for constructing such a pair for a convex $n$-gon.

Note that we do not construct the pair with the optimal quality for a given convex $n$-gon. Our proof seems to suggest that the best approximating pair $(r, R)$ of rectangles might be found in roughly linear time by sweeping from $\alpha=0$ to $\alpha=\frac{\pi}{2}$, maintaining $R(\alpha)$ and its largest homothetic copy $r(\alpha)$ inside $C$ during the process. However, a simpler version of this problem is already more difficult than it seems. If, instead of $R(\alpha)$, we take a rotated copy $T(\alpha)$ of a fixed triangle $T$, the vertices of the homothetic inner copy $t(\alpha)$ do not necessarily move monotonically on $C$. In fact, the maximum number of events during the sweep is $\Theta\left(n^{2}\right)$; see Waupotitsch [W88]. The fastest known algorithm which finds the largest similar copy of a given triangle inside a convex $n$-gon $C$ takes $O\left(n^{2} \log ^{2} n\right)$ time and is due to Sharir and Toledo [ST94]. Therefore, computing the best approximating pair of rectangles in less than roughly $O\left(n^{2}\right)$ time seems to be difficult.

The generalization of Theorem 6 to higher-dimensional boxes is another open question.

\section{References}

[AHS95] H. Alt, D. Hsu, and J. Snoeyink. Computing the largest inscribed isothetic rectangle. In Proc. 7th Canadian Conf. Comput. Geom., Université Laval, Québec, August 1995, pp. 67-72.

[A94] N. Amenta. Helly-type theorems and generalized linear programming. Discr. Comput. Geom. 12 (1994), 241-261.

[FMR ${ }^{+}$90] R. Fleischer, K. Mehlhorn, G. Rote, E. Welzl, and C. K. Yap. Simultaneous inner and outer approximation of shapes. Algorithmica 8 (1992), $365-389$. 
[KS95] D. Kirkpatrick and J. Snoeyink. Tentative prune-and-search for computing fixed-points with applications to geometric computation. Fundamenta Informatic 222 (1995), 353-370.

[Joh48] F. John. Extremum problems with inequalities as subsidiary conditions. In Studies and essays presented to R. Courant on his 60th Birthday, January 8, 1948, Interscience Publ., New York, 1948, pp. 187-204.

[Las89] M. Lassak. Approximation of plane convex bodies by centrally symmetric bodies. J. London Math. Soc. (2) 40 (1989), 369-377.

[Las92] M. Lassak. Approximation of convex bodies by triangles. Proc. Amer. Math. Soc. 115 (1992), 207-210.

[Las93] M. Lassak. Approximation of convex bodies by rectangles. Geom. Dedicata 47 (1993), 111-117.

[Lei59] K. Leichtweiß. Über die affine Exzentrizität konvexer Körper. Arch. Math. (Basel) 10 (1959), 187-199.

[PS51] G. Pólya and G. Szegő. Isoperimetric inequalities in mathematical physics. Ann. of Math. Studies, Vol. 27, Princeton Univ. Press, 1951.

[SFR ${ }^{+90] ~ O . ~ S c h w a r z k o p f, ~ U . ~ F u c h s, ~ G . ~ R o t e, ~ a n d ~ E . ~ W e l z l . ~ A p p r o x i m a t i o n ~ o f ~}$ convex figures by pairs of rectangles. In Proc. 7th Ann. Symp. Theoret. Aspects Computer Sci. (STACS'90), Rouen, February 1990. Lecture Notes in Computer Science 415, Springer-Verlag, 1990, pp. 240-249.

[ST94] M. Sharir and S. Toledo. Extremal polygon containment problems. Comput. Geom. Theory Appl. 4 (1994), 99-118.

[W88] R. Waupotitsch. Rotating a convex polygon in a convex polygon. Diplomarbeit (M. Sc. thesis), Techn. Univ. Graz, Institut für Informationsverarbeitung, 1988, 51 pp. 